\title{
Simulating metagenomic stable isotope probing datasets with MetaSIPSim
}

\author{
Samuel E. Barnett and Daniel H. Buckley ${ }^{*}$ (D)
}

\begin{abstract}
Background: DNA-stable isotope probing (DNA-SIP) links microorganisms to their in-situ function in diverse environmental samples. Combining DNA-SIP and metagenomics (metagenomic-SIP) allows us to link genomes from complex communities to their specific functions and improves the assembly and binning of these targeted genomes. However, empirical development of metagenomic-SIP methods is hindered by the complexity and cost of these studies. We developed a toolkit, 'MetaSIPSim,' to simulate sequencing read libraries for metagenomic-SIP experiments. MetaSIPSim is intended to generate datasets for method development and testing. To this end, we used MetaSIPSim generated data to demonstrate the advantages of metagenomic-SIP over a conventional shotgun metagenomic sequencing experiment.

Results: Through simulation we show that metagenomic-SIP improves the assembly and binning of isotopically labeled genomes relative to a conventional metagenomic approach. Improvements were dependent on experimental parameters and on sequencing depth. Community level $\mathrm{G}+\mathrm{C}$ content impacted the assembly of labeled genomes and subsequent binning, where high community $G+C$ generally reduced the benefits of metagenomic-SIP. Furthermore, when a high proportion of the community is isotopically labeled, the benefits of metagenomic-SIP decline. Finally, the choice of gradient fractions to sequence greatly influences method performance.

Conclusions: Metagenomic-SIP is a valuable method for recovering isotopically labeled genomes from complex communities. We show that metagenomic-SIP performance depends on optimization of experimental parameters. MetaSIPSim allows for simulation of metagenomic-SIP datasets which facilitates the optimization and development of metagenomic-SIP experiments and analytical approaches for dealing with these data.
\end{abstract}

Keywords: Stable isotope probing, SIP, Metagenomics, Simulation

\section{Background}

DNA-Stable isotope probing (DNA-SIP) is a powerful tool for linking uncultured microorganisms to their function within environmental samples [1]. DNA-SIP has applications in a wide range of areas including biogeochemistry [2-9], biodegradation [10-14], and ecological interactions $[15,16]$. In the past few years, methods such as high-resolution SIP (HR-SIP) [2] and quantitative SIP (qSIP) [17], have been developed to analyze amplicon sequencing data from DNA-SIP experiments. DNA-SIP has also been combined with metagenomic sequencing (metagenomic-SIP) to link in situ metabolic activity to genome composition $[13,18,19]$.

\footnotetext{
* Correspondence: dhb28@cornell.edu

School of Integrative Plant Science, Cornell University, Bradfield Hall, room 705, 306 Tower Rd, Ithaca, NY 14853, USA
}

Metagenomic-SIP is believed to improve the recovery of metagenome-assembled genomes (MAGs) from ${ }^{13} \mathrm{C}$-labeled organisms [20, 21]. Unfortunately, validation and improvement of metagenomic-SIP methods and analytical tools have largely been hindered by the difficulty and cost of these experiments $[14,20]$.

A recently developed open-source toolkit, SIPSim [22], enables in-silico simulation of amplicon sequencing data from DNA-SIP experiments (i.e. species abundance tables). SIPSim has been used to compare various DNASIP analysis methods [22] and is useful for testing the design of DNA-SIP experiments, but does not simulate sequencing read libraries and therefore is of limited use for development and testing of metagenomic-SIP methods. From here on, we will use metagenomic-SIP to refer to shotgun metagenomic sequencing from a DNA-

(c) The Author(s). 2020 Open Access This article is distributed under the terms of the Creative Commons Attribution 4.0 International License (http://creativecommons.org/licenses/by/4.0/), which permits unrestricted use, distribution, and 
SIP experiment. Here, we present a newly developed toolkit, MetaSIPSim, to simulate metagenomic-SIP datasets. MetaSIPSim generates sequencing read libraries in FASTA or FASTQ format such as those generated in a metagenomic-SIP experiment. MetaSIPSim is freely available on Github (https://github.com/seb369/MetaSIPSim). While the same basic principles of DNA-SIP simulation are used by both MetaSIPSim and SIPSim, the implementation of the simulations are significantly different. SIPSim uses kernel density estimates to determine the distribution of the reference genomes across a buoyant density (BD) gradient, without maintaining identifiable information of genome fragments that would be needed to generate sequencing reads. MetaSIPSim on the other hand simulates the abundance of individual genome fragments within a recovered BD fraction and adjusts this abundance based on isotope incorporation. By maintaining genome fragment identity, MetaSIPSim can simulate next generation sequencing reads from the individual fragments. At this time, there are no other tools publicly available that generate simulated metagenomic-SIP read libraries. Our tool will allow researchers to rapidly and inexpensively test experimental parameters and develop analytical tools which will advance the sophistication and accuracy of metagenomic-SIP experiments. SIP approaches are increasingly popular in environmental microbiology.

We demonstrated the utility of MetaSIPSim by assessing whether the coverage, assembly, and MAG binning of target ${ }^{13} \mathrm{C}$-labeled genomes were improved by metagenomicSIP relative to a conventional shotgun metagenomic approach. We hypothesized that the enhanced performance of metagenomic-SIP relative to shotgun metagenomics depends on sequencing depth (i.e. number of reads recovered). To test this hypothesis, we ran all simulations at both $5,000,000$ reads $(5 \mathrm{M})$ and $10,000,000$ reads $(10 \mathrm{M})$. We predicted that the benefits of metagenomic-SIP for assembly and binning would be greatest when sequencing depth is lower (i.e. in $5 \mathrm{M}$ ). Furthermore, we hypothesized that the performance of metagenomic-SIP varies with the guanine and cytosine $(G+C)$ content of whole community DNA. $G+C$ content determines where a DNA fragment localizes in a buoyant density (BD) gradient [23, 24]. Variation in individual genome $\mathrm{G}+\mathrm{C}$ in complex communities has been shown to affect measurements of isotope incorporation in DNA-SIP experiments, such that unlabeled high G + C DNA can potentially co-migrate with labeled DNA of low $\mathrm{G}+\mathrm{C}$ content $[22,25]$. In addition, genomic $\mathrm{G}+\mathrm{C}$ content is not uniform within individual genomes and the average $\mathrm{G}+\mathrm{C}$ content of a genome fragment will differ from the average $\mathrm{G}+\mathrm{C}$ content of its source genome with variance a function of fragment length. It is unclear the extent to which variation in community level $\mathrm{G}+\mathrm{C}$ content, and intragenomic variation in $\mathrm{G}+\mathrm{C}$ content, affects metagenome-SIP analyses. To test the hypothesis that community-level genomic
$\mathrm{G}+\mathrm{C}$ content affects metagenomics-SIP performance, we simulated metagenomic-SIP experiments using communities that differ in $\mathrm{G}+\mathrm{C}$ distribution (low, medium, and high). We predicted that read coverage, metagenome assembly and MAG bin quality would vary with community $\mathrm{G}+\mathrm{C}$ content resulting in lower performance for high $\mathrm{G}+\mathrm{C}$ communities.

\section{Implementation}

MetaSIPSim has been tested on Ubuntu 16.04.4 and Mac OSX 10.12.6 operating systems running python 2.7. All dependencies and their development versions are provided in Additional file 1: Table S1. MetaSIPSim can run with parallel processes to reduce running time. MetaSIPSim can be memory intensive, depending mostly on the number of reference sequences, reference sequence size, and number of reads generated. It is recommended to do a test run to make sure that the local system has enough RAM to handle a desired simulation.

\section{Simulation procedure}

The input to MetaSIPSim is a configuration file with all parameters discussed below as well as input and output paths. One input is the directory containing the reference sequences. All reference sequences must be in FASTA format. A reference can be a whole genome, scaffolds, or contigs, but each reference must be in a separate FASTA file. If a reference is composed of multiple scaffolds, chromosomes, or plasmids, its file should be in multi-FASTA format. All scaffolds within this file will be processed as a single reference. A diagram of the simulation procedure is found in Fig. 1. The first step of the simulation is to fragment each reference into discrete sequence segments termed 'fragments.' Fragment size is based on a user provided distribution. The fragmentation process simulates DNA fragmentation patterns occurring during DNA extraction, such as from bead beating. Fragment size distributions can be of uniform, normal, truncated-normal, or skewed-normal distribution, which should be chosen based on empirical evidence from a user's own extraction methods. The fragmentation process is repeated several times such that each reference has a fragment coverage designated by the user to get a diverse sample of fragments for each reference.

MetaSIPSim has the capacity to perform two simulation modes. The first simulation mode is the single BD window method,' similar to heavy-SIP [22], sequencing a single gradient window using BD boundaries $\left(\rho_{\max }, \rho_{\max }\right)$ defined by the user. Most published metagenomic-SIP studies to date have employed this 'heavy-SIP method.' The second simulation mode treats each density gradient fraction independently, similar to HR-SIP [2], in which multiple fractions spanning the gradient are sequenced individually. For this fractionbased simulation mode, the simulation is performed independently for each fraction, with the individual fraction $\mathrm{BD}$ 
Simulated community

- Species abundance table

- Incorporatoridentification table



\section{Fragmentation}

- Fragment length from supplied distribution

- Process repeated until fragment coverage

- Each fragmentabundance $=\alpha$

Fragment abundance in gradient window

- New fragment abundance $\left(\alpha_{f}\right)$ calculated from on lumen and DBL portions (equations 1-12)

\section{Calculate read abundances}

- Read abundance $\left(\alpha_{r}\right)$ calculated from equation 13 .

Calculate readrelative abundances

- Relative abundance for each read is equal to $a_{r}$ divided by sum of the abundance of all other reads

\section{Generate reads \\ - Number of reads from fragment randomly assigned weighted by relative abundances of the reads}

Output read sequences to fasta file

Fig. 1 Diagram of the simulation procedure

boundaries defining the gradient window $\left(\rho_{\max }, \rho_{\max }\right)$. The fraction-based simulation mode uses the same reference fragments in all individual simulations. All variables used in the following equations are summarized in Table 1.

Following reference sequence fragmentation, the abundance of each fragment within the gradient window is estimated. The initial fragment abundance $(\alpha)$ is equal to the relative abundance of the parent reference, provided in a user supplied community composition table. The abundance of each fragment in the gradient window is then determined as a function of fragment BD characteristics. The theoretical BD for the fragment $\left(\rho_{t}\right)$ is calculated from the $G+C$ of the fragment (Eq. 1) [26]:

$$
\rho_{t}=0.098(G+C)+1.66
$$

The theoretical $\mathrm{BD}$ is then adjusted for isotopic labeling based on the atom \% excess $(A)$ assigned to parent reference. The atom \% excess for an isotopically labeled fragment is randomly generated from a normal distribution, with the mean and standard deviation for each parent reference supplied in a user supplied incorporator identification table. With this setup, users can set different incorporators to varying levels of isotope labeling. If the fragment is from an unlabeled reference, the atom \% excess is 0 . The mean BD ( $\rho)$ of each fragment is calculated as such (Eq. 2) [22]:

$$
\rho=\rho_{t}+(A * \delta)
$$

Here, $\delta$ is the increase in BD of DNA if the atom \% excess is $100 \%$ (i.e. if the DNA was fully isotopically labeled). $\delta$ differs by isotope where $\delta=0.036$ for ${ }^{13} \mathrm{C}$ and $\delta=0.016$ for ${ }^{15} \mathrm{~N}[24]$.

Next, it is necessary to calculate 'DNA smearing' due to diffusive boundary layer effects during the deceleration 
Table 1 Descriptions of variables used in simulation equations

\begin{tabular}{|c|c|}
\hline Variable & Description \\
\hline$G+C$ & Proportion of DNA that is guanine or cytosine \\
\hline$\rho_{t}$ & Theoretical BD of a fragment based on fragment $\mathrm{G}+\mathrm{C}$ \\
\hline$\rho$ & $\mathrm{BD}$ of fragment after accounting for isotopic labeling \\
\hline$\sigma$ & Standard deviation of fragment BD $(\rho)$ \\
\hline$\rho_{\min }$ & Minimum BD of window to be sequenced \\
\hline$\rho_{\max }$ & Maximum BD of window to be sequenced \\
\hline$\rho_{D B L \min }$ & Minimum $B D$ of the $D B L$ for a fragment \\
\hline$\rho_{D B L \min }$ & Maximum $\mathrm{BD}$ of the $\mathrm{DBL}$ for a fragment \\
\hline A & Atom $\%$ excess of the fragment \\
\hline$\delta$ & Increase in DNA BD if atom \% excess is $100 \%\left({ }^{13} \mathrm{C}: 0.036,{ }^{15} \mathrm{~N}: 0.016\right)$ \\
\hline$p_{D B L}$ & $\begin{array}{l}\text { Proportion of DNA found in the diffusive boundary layer. } 1-p_{D B L} \text { is the } \\
\text { proportion of DNA found in the lumen of the tube }\end{array}$ \\
\hline$p_{L R}$ & Proportion of the fragment lumen population recovered in the BD window \\
\hline$r_{t}$ & Radius of the ultracentrifuge tube \\
\hline$r_{\min }$ & Minimum distance from the axis of rotation to the tube \\
\hline$r_{\max }$ & Maximum distance from the axis of rotation to the tube \\
\hline$x$ & Fragment distance from the axis of rotation at equilibrium \\
\hline$x_{\min }$ & Minimum position on tube of the $D B L$ range for a fragment \\
\hline$x_{\max }$ & Maximum position on tube of the DBL range for a fragment \\
\hline a & Abundance of the genome/fragment in the sample \\
\hline$a_{L}$ & Abundance of the fragment in the lumen of the tube within the BD \\
\hline$a_{D B L}$ & Abundance of a fragment in the $\mathrm{DBL}$ within the $\mathrm{BD}$ window \\
\hline$a_{f}$ & Abundance of the fragment in the $\mathrm{BD}$ window \\
\hline$a_{r}$ & Abundance of reads from the fragment \\
\hline 1 & Length of the fragment in base pairs \\
\hline$I_{r}$ & Read length in base pairs \\
\hline$R$ & Universal gas constant $(8.314 \mathrm{~J} / \mathrm{molK})$ \\
\hline$T$ & Temperature in kelvin \\
\hline$\beta$ & Proportionality constant of aqueous cesium chloride $\left(1.14 \times 10^{9}\right)$ \\
\hline G & Buoyancy factor $\left(7.87 \times 10^{-10}\right)$ \\
\hline$M_{c}$ & Mean molar weight of standard nucleotide base pair in cesium chloride solution (882) \\
\hline$D$ & Average density of the gradient solution \\
\hline$\omega$ & Angular velocity of centrifugation \\
\hline 1 & Isoconcentration point \\
\hline$\theta$ & Angle of the tube relative to the axis of rotation in radians \\
\hline
\end{tabular}

stage of ultracentrifugation when gradient reorientation occurs (Additional file 1: Figure S1) [22]. Most DNA is present in the 'lumen' of the centrifuge tube, which is defined as all DNA distant from and not interacting with the ultracentrifuge tube walls (Additional file 1: Figure S1). When the gradient reorients during deceleration, interactions with tube walls causes a thin layer of gradient solution containing DNA to be trapped within the diffusive boundary layer (DBL) along the tube walls [22]. The DBL does not move with the lumen-DNA during reorientation and, during fractionation, this unequilibrated 'DBL-DNA' will contaminate fractions with which it intersects (Additional file 1: Figure S1). The proportion of DNA in the DBL is minor compared to the lumen DNA, but is readily detected with high throughput sequencing approaches [22]. For simplicity in the simulation, the proportion of DNA found in the DBL $\left(p_{D B L}\right)$ relative to the total DNA concentration is provided by the user. Empirical studies are needed to determine the equations and experimental properties governing the ratio of DBL-DNA to lumen DNA. 
Within the tube lumen, due to diffusion, a fragment will be normally distributed around the isotope adjusted BD ( $\rho$ ) and standard deviation (6; Eq. 3; Additional file 1: Figure S1) [27]:

$$
\sigma=\sqrt{\frac{\rho R T}{\beta^{2} G M_{c} l}}
$$

Where $R$ is the universal gas constant $(8.314 \mathrm{~J} / \mathrm{molK}), T$ is the temperature in Kelvin, $\beta$ is the proportionality constant of aqueous cesium chloride $\left(1.14 \times 10^{9}\right)$ [28], $G$ is a buoyancy factor $\left(7.87 \times 10^{-10}\right)[29], M_{c}$ is the mean molecular weight of a standard nucleotide base pair in cesium chloride solution $(882 \mathrm{~g} / \mathrm{mol})$ [29], and $l$ is the length of the fragment in base pairs. The proportion of the fragment lumenpopulation recovered in the $\mathrm{BD}$ window $\left(p_{L R}\right)$ is then calculated from the cumulative density function of the normal distribution with mean $\rho$ and standard deviation $\sigma$ and bounded by the maximum and minimum buoyant densities of the gradient window $\left(\rho_{\max }, \rho_{\max }\right.$; Additional file 1: Figure S1). Thus, the abundance of the lumen-fragment $\left(\alpha_{L}\right)$ in the window is the total lumen abundance multiplied by this proportion recovered in the window (Eq. 4).

$$
\alpha_{L}=\alpha\left(1-p_{D B L}\right) \times p_{L R}
$$

To calculate the abundance of the DBL-fragment recovered in the gradient window, the range of buoyant densities that the DBL-fragment is contaminating is determined. First, the distance from the axis of rotation that the fragment will be found $(x)$ at equilibrium is calculated (Eq. 5) [24]:

$$
\begin{aligned}
& x=\sqrt{\left(\frac{2 \beta(\rho-D)}{\omega^{2}}\right)+I^{2}} \\
& I=\sqrt{\frac{r_{\text {min }}^{2}+r_{\text {min }} r_{\max }+r_{\text {max }}{ }^{2}}{3}}
\end{aligned}
$$

Here, $D$ is the average density of the gradient in $\mathrm{g} / \mathrm{ml}$, $\omega$ is the angular velocity of centrifugation in $\mathrm{rad} / \mathrm{s}$, and $I$ is the isoconcentration point (Eq. 6 [24]). $r_{\min }$ and $r_{\max }$ are the minimum and maximum distances from the axis of rotation to the tube (Additional file 1: Figure S1). From this, the minimum position $\left(x_{\text {min }}\right)$ of the DBL range along the ultracentrifuge tube can be calculated both if found in the middle, cylindrical section (Eq. 7) or the bottom, rounded section (Eq. 8) of the ultracentrifuge tube (Additional file 1: Figure S1) [22].

$$
\begin{aligned}
& x_{\text {min }}=r_{t}+\frac{r_{\text {max }}-r_{t} \cos \theta-r_{t}-x}{\sin \theta} \\
& x_{\text {min }}=r_{t}-r_{t} \cos \left(\theta-\sin ^{-1}\left(\frac{x-r_{\text {max }}+r_{t}}{r_{t}}\right)\right)
\end{aligned}
$$

Here, $\theta$ is the angle of the tube relative to the axis of rotation in radians, $r_{t}$ is the radius of the ultracentrifuge tube. Similarly, the maximum positions $\left(x_{\max }\right)$ of the DBL range is calculated whether in the middle, cylindrical section (Eq. 9) or the bottom, rounded section (Eq. 10) of the ultracentrifuge tube [22].

$$
\begin{aligned}
& x_{\text {max }}=r_{t}+\frac{r_{\text {max }}+r_{t} \cos \theta-r_{t}-x}{\sin \theta} \\
& x_{\text {max }}=r_{t}-r_{t} \cos \left(\theta-\pi+\sin ^{-1}\left(\frac{x-r_{\text {max }}+r_{t}}{r_{t}}\right)\right)
\end{aligned}
$$

Then the maximum and minimum positions are converted into BD limits $\left(\rho_{D B L \text { min }}\right.$ and $\left.\rho_{D B L \max }\right)$ from a table generated with a model gradient. Model gradients are generated as in SIPSim [22].

The abundance of the DBL-fragment $\left(\alpha_{D B L}\right)$ recovered in the gradient window is the proportion of the $\mathrm{DBL} B D$ range covered by the window then multiplied by the total abundance of the fragment DBL-population (Eq. 11).

$$
\alpha_{D B L}=\frac{\rho_{\max }-\rho_{\min }}{\rho_{D B L \max }-\rho_{D B L \min }}\left(\alpha \times p_{D B L}\right)
$$

From the previous calculations, the abundance of a fragment recovered in the sequenced BD window $\left(\alpha_{f}\right)$ is simply the sum of the abundances of lumen and DBL fragments (Eq. 12).

$$
\alpha_{f}=\alpha_{L}+\alpha_{D B L}
$$

Next, sequencing reads are generated from the fragments. To do this, the estimated relative abundance of each potential read $\left(\alpha_{r}\right)$ derived from the fragment is determined (Eq. 13) based on the lengths of the fragment $\left(l_{f}\right)$ and of the reads $\left(l_{r}\right)$.

$$
\alpha_{r}=\frac{\alpha_{f} * l_{f}}{2 l_{r}}
$$

Common read lengths for Illumina sequencing chemistry include $125,150,250$, and $300 \mathrm{bp}$. The initial read abundance is transformed into a relative abundance by dividing by the sum of all read abundances across the fragment. Then the number of reads to be recovered from each fragment is assigned randomly, weighted by the relative abundance of reads within each fragment. Each paired end read from each fragment is then generated by randomly selecting forward and reverse read starting points based on the read length and insert size. Insert size is randomly generated for each read based on a normal distribution with mean and standard deviation provided by the user. Forward or reverse assignment is random. Finally, read sequences are retrieved from the original reference sequence based on coordinates which have been propagated from fragment to sequencing read. Reverse reads are converted to the reverse compliment. 
All final forward and reverse read sequences are written out to two multi-FASTA files with paired unique identifiers.

To compare metagenomic-SIP data to a standard shotgun metagenomic dataset, MetaSIPSim includes a script for generating bulk community metagenomes with the same references and parameters as the metagenomic-SIP simulation. This script functions similarly to the metagenomic-SIP simulator, using the same input configuration file. However, in this case the abundance of each simulated fragment $\left(\alpha_{f}\right)$ is equal to the reference abundance $(\alpha)$, without adjustments for gradient fractionation. The FASTA output generated by MetaSIPSim has no sequencing errors or quality score information associated with high throughput sequencing datasets. We have included a script that includes modified functions from InSilicoSeq [30] to convert the FASTA file output into FASTQ format by simulating sequencing errors and quality scores. The conversion uses an error model included in the InSilicoSeq installation or created by the user. The error models are sequencing platform specific, with options for Illumina MiSeq, HiSeq, or NovaSeq. Unlike the original InSilicoSeq implementation, the MetaSIPSim script does not add gaps to sequences.

While most previous metagenomic-SIP studies have used Illumina high-throughput sequencing technologies, other sequencing methods may be of interest to researchers. Long read sequencing from Pacific Biosciences (PacBio) singlemolecule real-time sequencing or Oxford Nanopore Technologies, often in conjunction with high throughput technologies, can improve metagenome assembly [31, 32]. PacBio specifically has been used in conjunction with Illumina HiSeq sequencing for metagenomic-SIP [33]. MetaSIPSim does not explicitly simulate read generation from these alternative sequencing methods, however modifications or additions to this toolkit can allow sequencing read simulations across a large variety of sequencing technologies. A roadmap for these extensions can be found in the supplementary materials.

\section{Validation}

We validated the ability of MetaSIPSim to simulate the distribution of both isotopically labeled and unlabeled genomic DNA across a $\mathrm{CsCl}$ gradient against three published DNA-SIP studies [25, 34, 35]. These studies used bacterial isolates grown on ${ }^{13} \mathrm{C}$-labeled, ${ }^{15} \mathrm{~N}$-labeled, or unlabeled substrates (Table 2). For each study, we simulated fragments across multiple $\mathrm{CsCl}$ gradient fractions. Fraction BD boundaries were estimated based on the reported average fraction BD. Gradient parameters were taken from those reported (Additional file 1: Table S2). We used a uniform atom \% excess of $100 \%$ as these studies used pure cultures and fully labeled substrates.
The code for validation simulations available at https:// github.com/seb369/MetaSIPSim/validation/.

All three studies measured the amount of genomic DNA of an isolate recovered across fractions, normalized to the fraction with the highest DNA concentration. To approximate this value, within each fraction, we multiplied the abundance of each fragment by its length, then summed this base pair count across all fragments get a quantity of genomic DNA. We then normalized to the fraction with the greatest quantity of DNA to get the gradient profile. Empirical distributions were estimated from the published figures using Engage Digitizer version 6.0 [36]. We ran simulations with SIPSim [22] using the same parameters as an additional comparison.

\section{Case study: assessment of improved metagenomes using metagenomic-SIP}

We used MetaSIPSim to assess whether metagenomicSIP improves coverage, assembly, and binning of labeled bacterial genomes compared to conventional shotgun metagenome sequencing. The simulated SIP experiment was based on a ${ }^{13} \mathrm{C}$, heavy window metagenomic-SIP experiment. A diagram of the experimental design is found in Additional file 1: Figure S2. A total of 1542 reference genomes were downloaded from the NCBI RefSeq database [37] on January 25, 2019. Genomes that were related at the species level, based on reported taxonomy, were pruned such that a single genome was present per species. The remaining 1491 reference genomes had a bimodal distribution of $\mathrm{G}+\mathrm{C}$, with peaks around 40 and 65\% (Additional file 1: Figure S3). To get a representative set of reference genomes with a relatively uniform $\mathrm{G}+\mathrm{C}$ distribution for sampling, we sampled 20 genomes from each integer between 20 and $75 \% \mathrm{G}+\mathrm{C}$ after rounding $\mathrm{G}+\mathrm{C}$ values to the nearest integer. If 20 or fewer genomes were present at a given $\mathrm{G}+\mathrm{C}$ integer, then all genomes with that $\mathrm{G}+\mathrm{C}$ value were selected. From this subset of genomes, we then randomly subsetted 500 genomes to meet $\mathrm{G}+\mathrm{C}$ content criteria for the reference sets: lowGC $(40 \%)$, medGC (50\%), and highGC (60\%). Genome selection was weighted by the absolute value of the distance of each genome's $\mathrm{G}+\mathrm{C}$ to the target community $\mathrm{G}+\mathrm{C}$ (Additional file 1: Figure S4).

From each reference set, we generated six replicate synthetic communities with randomized species abundance distributions (Additional file 1: Figure S5). These replicate communities represent the variation one would expect from a SIP experiment with biological replicates, such as independent microcosms. The compositions of each replicate community was generated using the communities function from SIPSim [22] with all genomes present (richness $=1$ ) in each replicate, with a lognormal distribution, a mean relative abundance of $2.0 \%$, and standard deviation of 0.8 . All genomes from the 
Table 2 Studies used for validation of fragment abundance distributions including the isolates reported in the study, NCBI accessions used to download the genomes, and the stable isotopes simulated

\begin{tabular}{llll}
\hline Study & Isolates (NCBI accession) & Isotope(s) & Original publication figure \\
\hline Lueders et al. 2004 & Methanosarcina barkeri MS (CP009528.1) & $1 \mathrm{~A}$ \\
& Methylobacterium extorquens AM1 (NC_012808.1) & ${ }^{12} \mathrm{C}$ & 2 \\
Buckley et al. 2007 & Escherichia coli K12 (NC_000913.3) & ${ }^{14} \mathrm{~N},{ }^{15} \mathrm{~N}$ & \\
Wawrik et al. 2009 & Pseudomonas aeruginosa PAO1 (NC_002516.2) & ${ }^{14} \mathrm{~N}$ & $3 \mathrm{~B}$ \\
\hline
\end{tabular}

reference set were found in each community but $10 \%$ of abundance ranks were permuted, to provide more realistic variation between communities. Overall, we simulated a total of 18 communities. The first replicate community from each reference set was designated as the control, in which no genomes were isotopically labeled.

We randomly selected $20 \%$ of each reference set (i.e. 100 genomes) to be isotopically enriched 'incorporators.' To avoid a reference set with incorporators disproportionality weighted to either high or low abundance compared to the other sets, genomes were selected such that the mean abundance ranks of incorporators were similar across all three reference sets (Additional file 1: Figure S5). For each treatment community, 50 genomes from the incorporator set were randomly chosen to be labeled. This process was reiterated until all 100 incorporators were assigned to at least one treatment community. All incorporators had a mean atom $\%$ excess of ${ }^{13} \mathrm{C}$ of $90 \%$ and standard deviation of 5 . This experimental design, with five treatment and one control sample, where labeled genomes may vary between treatment samples represents a metagenomic-SIP experiment where different labeled substrates were supplied to enrich different populations within a community [2,38]. In this type of experiment organisms can be labeled under more than one treatment and one unlabeled replicate community can act as a control for multiple treatments. $\mathrm{CsCl}$ gradients were simulated for each sample using the gradient fractions function from SIPSim [22] with minimum and maximum buoyant densities of 1.675 and $1.771 \mathrm{~g} / \mathrm{ml}$.

We simulated both metagenomic-SIP and conventional shotgun metagenome reads from the synthetic communities using MetaSIPSim. SIP gradient parameters were derived from Pepe-Ranney et al. [2] (Additional file 1: Table S2). These parameters were used as they represent a standard DNA-SIP experiment used in a number of studies. For both types of simulations, we generated 5,000,000 $(5 \mathrm{M})$ and 10,000,000 $(10 \mathrm{M})$ paired end, 151 bp reads with an average insert size of $1000 \mathrm{bp}$ and standard deviation of 5. Sequencing errors and quality scores were added using the NovaSeq error model from InSilicoSeq [30]. We performed the following metagenome processing pipeline to assemble and bin contigs separately for each reference set, read depth, and metagenome simulation type: $(i)$ co- assembly was performed with the six read libraries, one per replicate community, using MEGAHIT version 1.1.3 [39] with default parameters, and (ii) contigs were binned with MetaBAT2 version 2.12.1 [40, 41] using default parameters. MetaBAT2 was chosen as it incorporates differential abundance binning, which takes advantage of the differential labeling of genomes across treatments [21]. All code for these simulations including metagenome data processing are available at https:/github.com/seb369/ MetaSIPSim/tree/master/case_study.

We assessed how well each reference genome was recovered in raw reads by separately mapping libraries to the references using BBMap version 37.10 [42]. Specifically, we used the genome coverage and the proportion of the genome completely mapped by reads as indicators. We assessed assembly quality through alignment of contigs to the reference genomes using MetaQUAST version 5.0.2 [43]. The metrics used to gauge the assembly quality were the proportion of each reference genome aligned to contigs and the NGA50 for each reference. Successful MAG binning was assessed by first determining which genome was most aligned to each MAG. The proportion of the reference recovered as a MAG was calculated as length of the genome aligned to the binned contigs divided by genome length. Sections of the reference genome where multiple contigs aligned were only counted once (i.e. contig overlaps). Bin contamination was calculated as the summed length of contig regions not aligned to the assigned reference divided by the total bin size. In all analyses, we compared assessment metrics between the metagenomic-SIP and the shotgun metagenomic datasets. All statistical analyses were performed in $\mathrm{R}$ version 3.4.4 [44] using the Wilcoxon signed rank tests.

We ran two follow up analyses based on trends observed with initial simulations. First, we tested whether the number of isotopically labeled genomes within each sample influenced metagenomic-SIP performance. This simulation was done using the lowGC reference set. Incorporators were randomly selected as before, however we selected 25 incorporators per treatment (from 50 total incorporators in the reference set) or 100 incorporators per treatment (from 200 in the reference set). All other community and simulation parameters were identical to the original simulations. Secondly, we tested whether the selection of the 
$\mathrm{BD}$ window to sequence influences metagenomic-SIP improvement. These simulations were done using the highGC reference set. All community and simulation parameters were identical as before except that the $\mathrm{BD}$ window to be sequenced was $1.70-1.75 \mathrm{~g} / \mathrm{ml}, 1.72-1.77 \mathrm{~g} / \mathrm{ml}$, or $1.75-1.79 \mathrm{~g} / \mathrm{ml}$. The BD range for the model gradients were also slightly extended to $1.67-1.80 \mathrm{~g} / \mathrm{ml}$ to account for the new window ranges. We simulated 5,000,000 reads for all follow up simulations. All metagenome processing and analyses were performed as previously described.

\section{Results}

\section{Implementation}

Simulations for the metagenomic-SIP case study took on average $98 \mathrm{~min}$ and $121 \mathrm{~min}$ for six replicate communities at 5,000,000 and 10,000,000 reads respectively, using 10 processors. The corresponding simulations for conventional shotgun sequencing took approximately $38 \mathrm{~min}$ and 56 min respectively. Fragment generation took up to 10 min of those runs, with all additional time required for generating reads for each of the six replicate communities (Additional file 1: Table S3). These processing times do not include generation of input files, conversion from FASTA to FASTQ formats, or any read processing or analysis. Overall the simulations used less than $20 \mathrm{~GB}$ of RAM on an Ubuntu 16.04.4 operating system.

\section{Validation}

We simulated the distribution of fragmented genomic DNA across $\mathrm{CsCl}$ gradients based on experimental procedures from three published studies (Table 2). We found that the peak genomic DNA density recovery from the MetaSIPSim and SIPSim simulations roughly matched the empirical results from Buckley et al. 2007 [25] and Wawrik et al. 2009 [35] (Fig. 2b and c) and the general distributions were similar across datasets for these studies. Variations in overall distributions are likely due in part to variability from experimental conditions and variations in genome composition between isolate and reference sequence strains. For the Lueders et al. 2004 [34] study, the peak genomic DNA density matched between MetaSIPSim and SIPSim, however these peaks were in adjacent $\mathrm{BD}$ fractions compared to the empirical data (Fig. 2a). This small difference may be due to variation in experimental parameters or methodology that were not simulated.

Future studies can examine the predictive power of MetaSIPSim across a wide variety of microbial community designs by utilizing synthetic microbial communities with known genomes, abundance profiles, and isotope incorporation patterns. In such a study, the known community information would allow MetaSIPSim to predict the sequencing output of a metagenomic-SIP experiment. These predictions can then be directly compared to empirical sequencing data from the synthetic community experiment.
The variation between simulated and empirical data can be quantified by measuring differences in genome coverage between the two datasets.

\section{Metagenomic-SIP improves recovery of isotopically labeled genomes in raw reads}

We used MetaSIPSim to assess the ability of metagenomicSIP to improve genome assembly and binning relative to shotgun metagenomics. Metagenomic-SIP should enrich for reads from isotopically labeled genomes (i.e. incorporators), so we first examined coverage and recovery of incorporator genomes in our raw simulated reads. As expected, we found that incorporators had greater coverage and were recovered more completely in the metagenomic-SIP simulation compared to paired conventional metagenomic libraries (Fig. 3a and c, Table 3). The difference in incorporator coverage and genome recovery between metagenomic-SIP and shotgun metagenomes were significantly greater than zero across all simulations (all $p$-values $<0.001$; Table 3 ). The increased coverage achieved by metagenomic-SIP was negatively correlated with community $\mathrm{G}+\mathrm{C}$. The increase in genome recovery in raw reads with metagenomic-SIP was highest for the medGC reference set. The increase in coverage with metagenomic-SIP for each incorporator was also strongly affected by the $\mathrm{G}+\mathrm{C}$ content of the target genome, where low $\mathrm{G}+\mathrm{C}$ genomes had the least fold difference in coverage with metagenomic-SIP compared to shotgun metagenomics (Additional file 1: Figure S6). The improvement that metagenomic-SIP provides in the recovery of labeled genomes within raw reads was also somewhat affected by genome $\mathrm{G}+\mathrm{C}$ but was more strongly driven by the relative abundance of target genomes in the community, with metagenomic-SIP providing the greatest benefit in recovering low abundance genomes (Additional file 1: Figure S7).

The number of incorporators had a minor impact on both labeled genome coverage and recovery within raw reads. The benefits of metagenomic-SIP relative to shotgun metagenomics were greatest when the number of incorporator genomes was low (Fig. 3b and d; Table 4). BD window position strongly influenced the coverage and recovery of targeted genomes within raw reads. Metagenomic-SIP analysis of a lighter BD window $(1.70-1.75 \mathrm{~g} / \mathrm{ml})$ showed little improvement relative to standard metagenomics sequencing. In contrast, metagenomic-SIP analysis of a heavier windows improved significantly the coverage and recovery of target genomes within raw reads $(1.72-1.77 \mathrm{~g} / \mathrm{ml}$ and $1.74-1.79 \mathrm{~g} /$ ml; Fig. 3b and d; Table 4). For both measures, there was a strong relationship between the incorporator $\mathrm{G}+\mathrm{C}$ and the $\mathrm{BD}$ of sequenced fractions. Metagenomic-SIP did not significantly improve coverage or recovery of raw reads from high $\mathrm{G}+\mathrm{C}$ genomes in the light BD window over shotgun metagenomics (Additional file 1: Figure S8). Conversely, metagenomic-SIP did little to improve read coverage or recovery of low $\mathrm{G}+\mathrm{C}$ genomes in the heavier $\mathrm{BD}$ window 
- empirical - - metaSIPSim … SIPSim

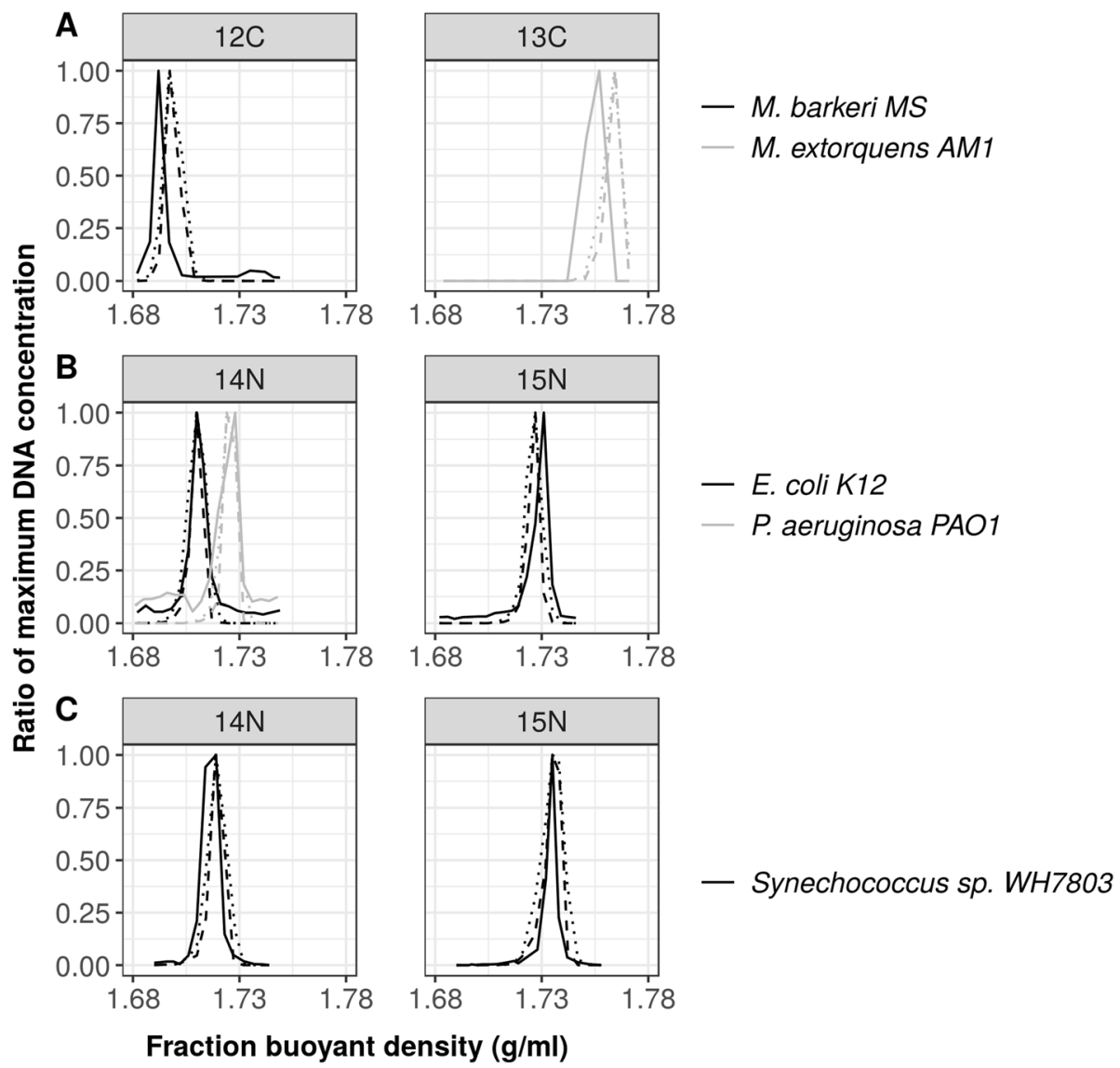

Fig. 2 Comparisons among MetaSIPSim and SIPSim simulated genomic DNA distribution across CsCl gradients and the empirical results from a Lueders et al. 2004 [34], b Buckley et al. 2007 [25], and c Wawrik et al. 2009 [35]

compared to the conventional approach (Additional file 1: Figure S9).

\section{Metagenomic-SIP improves assembly of isotopically labeled genomes}

We found that metagenomic-SIP improved assembly of isotopically labeled genomes over conventional shotgun metagenomics. When sequenced at relatively low depth, metagenomic-SIP allowed for a significantly greater proportion of each labeled genome to be assembled compared to the shotgun metagenomes in all three reference sets (Fig. 4a, Table 2). At high sequencing depth metagenomic-SIP improved target genome assembly in the low and medium $\mathrm{G}+\mathrm{C}$ reference sets but not in the high $\mathrm{G}+\mathrm{C}$ set. In all simulations, metagenomic-SIP improved quality of assembled contigs from labeled genomes, as measured by NGA50 (Fig. 4c, Table 3), and more incorporators were assembled at $\geq 50 \%$ completeness compared to the shotgun method (Additional file 1: Table S4). Considering the need to have at least $50 \%$ of the genome recovered to calculate the NGA50 from both
metagenomic-SIP and shotgun metagenomic assemblies, our NGA50 analysis was limited to a subset of relatively well assembled references. In all cases, but most notably with the high $\mathrm{G}+\mathrm{C}$ reference set, incorporators with higher $\mathrm{G}+\mathrm{C}$ were recovered to a higher proportion by assembled contigs with metagenomic-SIP over shotgun metagenomics (Additional file 1: Figure S10). Similarly, assembly improvement with metagenomic-SIP was greatest for low abundance incorporators, a trend most prominent with the lower $\mathrm{G}+\mathrm{C}$ genome set (Additional file 1: Figures $\mathrm{S} 10$ and S11).

The number of incorporators per sample influenced how metagenomic-SIP improved assembly of labeled genomes over shotgun metagenomics. Overall, both genome recovery in assembled contigs and NGA50 showed greater improvement with metagenomic-SIP when fewer genomes were labeled per sample. Metagenomic-SIP did not significantly increase the proportion of incorporator genomes recovered in contigs when we labeled 100 genomes per sample (Fig. $4 \mathrm{~b}$ and d, Table 4). The BD analysis window greatly impacted recovery and quality of 


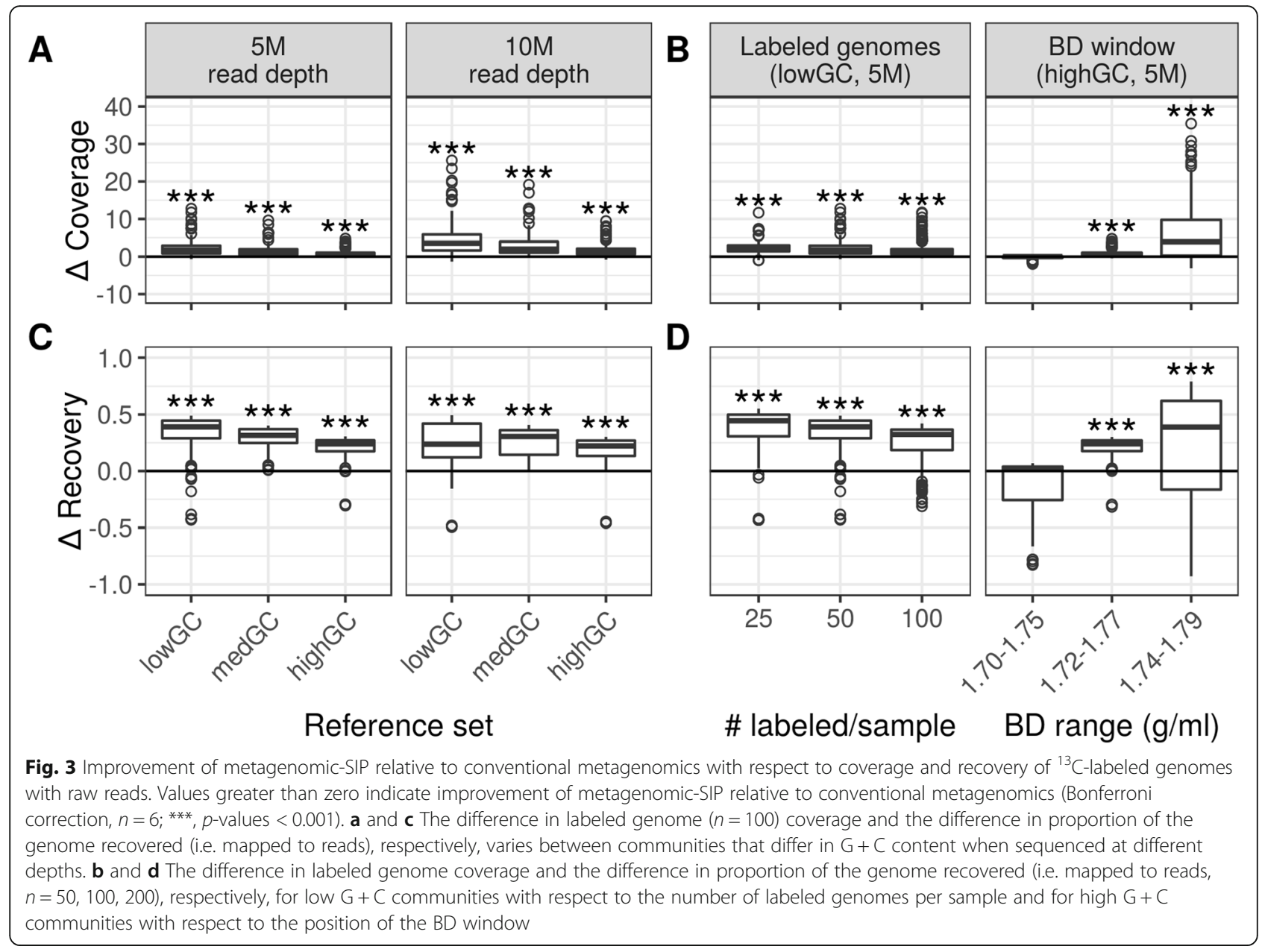

Table 3 Median difference $(\tilde{\Delta})$ in metagenome quality for labeled genomes between simulations of metagenomic-SIP and conventional shotgun metagenomic data. For all measures, except bin contamination, a difference greater than zero indicates that metagenomic-SIP improved metagenome quality relative to the conventional metagenomics approach. For bin contamination, a difference less than zero indicates improved metagenome quality with metagenomic-SIP. All statistical analyses were single sided, Wilcoxon signed rank tests with an alternate hypothesis of greater than zero, except for bin contamination which used an alternate hypothesis of less than zero. All $p$-values are adjusted for multiple comparisons (Bonferroni, $n=6$ )

\begin{tabular}{|c|c|c|c|c|c|c|c|}
\hline $\begin{array}{l}\text { Community } \\
\mathrm{G}+\mathrm{C}\end{array}$ & $\begin{array}{l}\text { Seq. } \\
\text { depth }\end{array}$ & $\begin{array}{l}\tilde{\Delta} \text { Read } \\
\text { coverage X } \\
(p \text {-value })\end{array}$ & $\begin{array}{l}\tilde{\Delta} \text { Proportion of genome } \\
\text { recovered in reads ( } p \text { - } \\
\text { value) }\end{array}$ & $\begin{array}{l}\tilde{\triangle} \text { Proportion of genome } \\
\text { recovered in contigs ( } p \text { - } \\
\text { value) }\end{array}$ & $\begin{array}{l}\tilde{\triangle} \text { NGA50 } \\
\text { bp }(p- \\
\text { value })\end{array}$ & $\begin{array}{l}\tilde{\triangle} \text { Proportion of genome } \\
\text { recovered in best bin ( } p \text { - } \\
\text { value) }\end{array}$ & $\begin{array}{l}\Delta \text { Proportion bin } \\
\text { contaminated ( } p \text { - } \\
\text { value) }\end{array}$ \\
\hline Low & $5 \mathrm{M}$ & $\begin{array}{l}1.769(< \\
0.001)\end{array}$ & $0.391(<0.001)$ & $0.052(<0.001)$ & $\begin{array}{l}19,672(< \\
0.001)\end{array}$ & $0.567(<0.001)$ & $-0.038(<0.001)$ \\
\hline Medium & & $\begin{array}{l}1.002(< \\
0.001)\end{array}$ & $0.315(<0.001)$ & $0.053(<0.001)$ & $\begin{array}{l}9251(< \\
0.001)\end{array}$ & $0.353(<0.001)$ & $-0.022(<0.001)$ \\
\hline High & & $\begin{array}{l}0.596(< \\
0.001)\end{array}$ & $0.240(<0.001)$ & 0.026 (NS) & $\begin{array}{l}3511(< \\
0.001)\end{array}$ & $0.366(<0.001)$ & $-0.010(0.001)$ \\
\hline Low & $10 \mathrm{M}$ & $\begin{array}{l}3.561(< \\
0.001)\end{array}$ & $0.237(<0.001)$ & $0.004(0.007)$ & $\begin{array}{l}11,636(< \\
0.001)\end{array}$ & $0.144(<0.001)$ & $-0.017(<0.001)$ \\
\hline Medium & & $\begin{array}{l}1.992(< \\
0.001)\end{array}$ & $0.306(<0.001)$ & $0.007(<0.001)$ & $\begin{array}{l}7554(< \\
0.001)\end{array}$ & $0.092(<0.001)$ & $-0.011(<0.001)$ \\
\hline High & & $\begin{array}{l}1.174(< \\
0.001)\end{array}$ & $0.222(<0.001)$ & 0.001 (NS) & $\begin{array}{l}7718(< \\
0.001)\end{array}$ & $0.134(<0.001)$ & $-0.009(<0.001)$ \\
\hline
\end{tabular}


Table 4 Median difference $(\tilde{\Delta})$ in metagenome quality measures for labeled genomes between simulations of metagenomic-SIP and conventional metagenomics. For all measures, except bin contamination, a difference greater than zero indicates that metagenomicSIP improved metagenome quality relative to conventional metagenomics. For bin contamination, a difference less than zero indicates improved metagenome quality with metagenomic-SIP. All statistical analyses were single sided, Wilcoxon signed rank tests with an alternate hypothesis of greater than zero, except for bin contamination which used an alternate hypothesis of less than zero. All $p$-values are adjusted for multiple comparisons (Bonferroni, $n=6$ )

\begin{tabular}{|c|c|c|c|c|c|c|c|c|}
\hline $\begin{array}{l}\text { Comm. } \\
G+C\end{array}$ & $\begin{array}{l}\text { \# Labeled } \\
\text { genomes } \\
\text { per sample }\end{array}$ & $\begin{array}{l}\text { Buoyant } \\
\text { Density } \\
\text { window } \\
\mathrm{g} / \mathrm{ml}\end{array}$ & $\begin{array}{l}\tilde{\Delta} \text { Read } \\
\text { coverage X ( } p- \\
\text { value) }\end{array}$ & $\begin{array}{l}\tilde{\Delta} \text { Proportion of } \\
\text { genome recovered } \\
\text { in reads ( } p \text {-value) }\end{array}$ & $\begin{array}{l}\tilde{\Delta} \text { Proportion of } \\
\text { genome recovered } \\
\text { in contigs ( } p \text {-value) }\end{array}$ & $\begin{array}{l}\tilde{\triangle} \text { NGA50 bp ( } p \text { - } \\
\text { value) }\end{array}$ & $\begin{array}{l}\tilde{\Delta} \text { Proportion of } \\
\text { genome recovered } \\
\text { in best bin ( } p \text {-value) }\end{array}$ & $\begin{array}{l}\tilde{\Delta} \text { Proportion } \\
\text { bin } \\
\text { contaminated } \\
(p \text {-value })\end{array}$ \\
\hline \multirow[t]{3}{*}{ Low } & 25 & $1.72-1.77$ & $2.202(<0.001)$ & $0.445(<0.001)$ & $0.058(0.027)$ & $18,409(<0.001)$ & $0.675(<0.001)$ & -0.007 (NS) \\
\hline & 50 & & $1.769(<0.001)$ & $0.391(<0.001)$ & $0.052(<0.001)$ & $19,672(<0.001)$ & $0.567(<0.001)$ & $\begin{array}{l}-0.038(< \\
0.001)\end{array}$ \\
\hline & 100 & & $1.188(<0.001)$ & $0.324(<0.001)$ & 0.006 (NS) & $7779(<0.001)$ & $0.337(<0.001)$ & $\begin{array}{l}-0.010(< \\
0.001)\end{array}$ \\
\hline \multirow[t]{3}{*}{ High } & 50 & $1.70-1.75$ & 0.018 (NS) & 0.013 (NS) & -0.032 (NS) & -212 (NS) & 0.019 (NS) & -0.001 (NS) \\
\hline & & $1.72-1.77$ & $0.589(<0.001)$ & $0.241(<0.001)$ & 0.026 (NS) & $3824(<0.001)$ & $0.330(<0.001)$ & $-0.017(0.006)$ \\
\hline & & $1.75-1.79$ & $3.935(<0.001)$ & $0.388(<0.001)$ & $0.102(0.013)$ & $47,027(<0.001)$ & $0.825(<0.001)$ & -0.012 (NS) \\
\hline
\end{tabular}

contigs in metagenomic-SIP. Metagenomic-SIP improved assembly the most for the heaviest BD window $(1.74-1.79 \mathrm{~g} / \mathrm{ml}$; Fig. $4 \mathrm{~b}$ and d, Table 4). The benefit of metagenomic-SIP over shotgun metagenomics was strongly influenced by the $\mathrm{G}+\mathrm{C}$ of the labeled genome interacting with the $\mathrm{BD}$ window. For high $\mathrm{G}+\mathrm{C}$ incorporators, using a light BD window $(1.70-1.75 \mathrm{~g} / \mathrm{ml})$ did little to improve assembly over shotgun metagenomics while for the low $G+C$ incorporators we saw little assembly improvement when using a heavy window (1.74$1.79 \mathrm{~g} / \mathrm{ml}$; Additional file 1: Figures S12 and S13).

\section{Metagenomic-SIP improves MAG binning of isotopically labeled genomes}

Finally, we found that metagenomic-SIP improved the binning of labeled genomes over conventional shotgun metagenomics. Across all simulations, more labeled genomes were recovered as MAGs with metagenomic-SIP compared to the conventional approach (Additional file 1: Table S4). We observed a high accuracy in MAG binning for both metagenomic-SIP and shotgun metagenomic datasets, with a minority of genomes divided or overlapping among multiple bins. Since some genomes were found in multiple bins, we examined binning quality in two ways. First, we used the single "best" bin for each labeled genome (i.e. the bin that covered the highest proportion of the reference genome). We found that a greater proportion of each labeled genome was recovered in the best bin when using metagenomic-SIP compared to shotgun metagenomics (Fig. 5a, Table 3). We further found that, for genomes that were successfully binned in both simulation types, metagenomic-SIP bins had less contamination from other genomes compared to the corresponding shotgun metagenomic bins (Fig. 5c, Table 2). Second, we combined the multiple bins identified as originating from the same reference genome. This multiple bin approach also showed improved labeled genome recovery and contamination relative to conventional shotgun metagenomics approaches (Additional file 1: Figure S14). Metagenomic-SIP improved binning the most at lower levels of sequencing depth.

We further found that both the number of incorporators per sample and the choice of the BD window to sequence influenced metagenomic-SIP binning improvement. We tested this using the single best bin approach. With more incorporators per sample, metagenomic-SIP did not improve labeled genome recovery within a bin as much as in simulations with fewer incorporators per sample. Metagenomic-SIP improved bin recovery the most when sequencing a heavier BD window (Fig. 5b, Table 4). Interestingly, moderate values for both number of incorporators per sample (50) and sequenced BD window (1.72-1.77 g/ $\mathrm{ml}$ ) showed the greatest improvement in bin contamination (Fig. 5d, Table 4).

\section{Discussion}

We developed the toolkit MetaSIPSim to simulate sequencing read datasets for metagenomic-SIP experiments. This is the first publicly available toolkit with this function and we believe it will be an important aid for development and testing of metagenomic-SIP experimental designs and analysis tools. As we presented here, MetaSIPSim can simulate an entire high-complexity metagenomic-SIP experiment. The toolkit employs many user-defined parameters that correspond to aspects of metagenomic-SIP experimental designs. By adjusting these parameters, researchers can use MetaSIPSim to optimize their methodologies prior to running their metagenomic-SIP experiments. Important methodological parameters that can be optimized in this way 


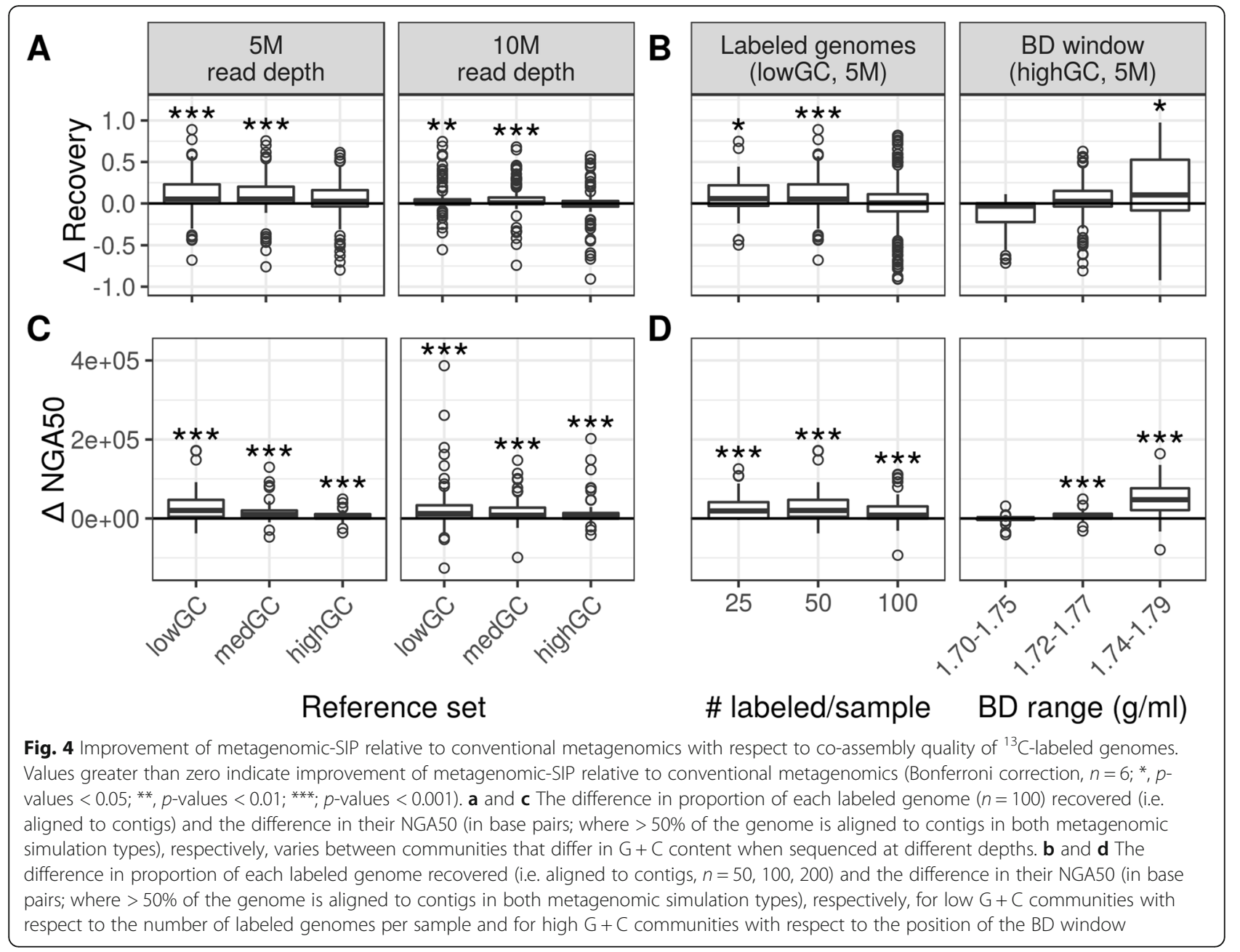

include buoyant density window or fraction sizes, $\mathrm{CsCl}$ densities, centrifugation velocities, sequencing depths, community complexities, and amounts of isotopic labeling. As MetaSIPSim runs quickly and has no reagent or sequencing costs, optimization can be run multiple times and in parallel for multiple different parameter values. We demonstrated this by running our case study in parallel at two different sequencing depths.

MetaSIPSim can be used in lieu of in vitro metagenomic-SIP experiments with mock communities to generate datasets for development of analytical pipelines, saving time and money. Datasets generated in silico with MetaSIPSim can be used to develop and test tools and pipelines specialized for assembly and binning isotopically labeled genomes. Most current metagenomic-SIP studies utilize a heavy window methodology. While this method may currently be the best option in most cases, with advancements to sequencing technologies and highthroughput methodologies, other methods similar to HRSIP [2] may be practical, utilizing multiple sequenced gradient fractions. HR-SIP-like methods may be useful for overcoming some of the previously described factors that interfere with metagenomic-SIP genome recovery such as community or incorporator $\mathrm{G}+\mathrm{C}$ but require new analytical tools. Simulated datasets are especially important for development of methods used to identify isotopically labeled contigs or MAGs. By having known reference genomes, atom \% excess values for each incorporator, and community profiles, developers can measure sensitivity and specificity of their tools. Further, simulations generated with MetaSIPSim are reproducible, allowing for comparisons between analysis tools.

MetaSIPSim can also be incorporated into a metagenomic-SIP analysis pipeline to identify labeled contigs, MAGs, or genomes. In such an approach, contigs assembled from metagenomic-SIP might be identified as either isotopically labeled or unlabeled by comparing their empirical coverage distributions across a $\mathrm{CsCl}$ gradient to simulated distributions produced with metaSIPSim. Incorporating simulated and actual read distributions in such analyses might provide an approach for identifying isotopically labeled DNA directly from metagenomics-SIP 


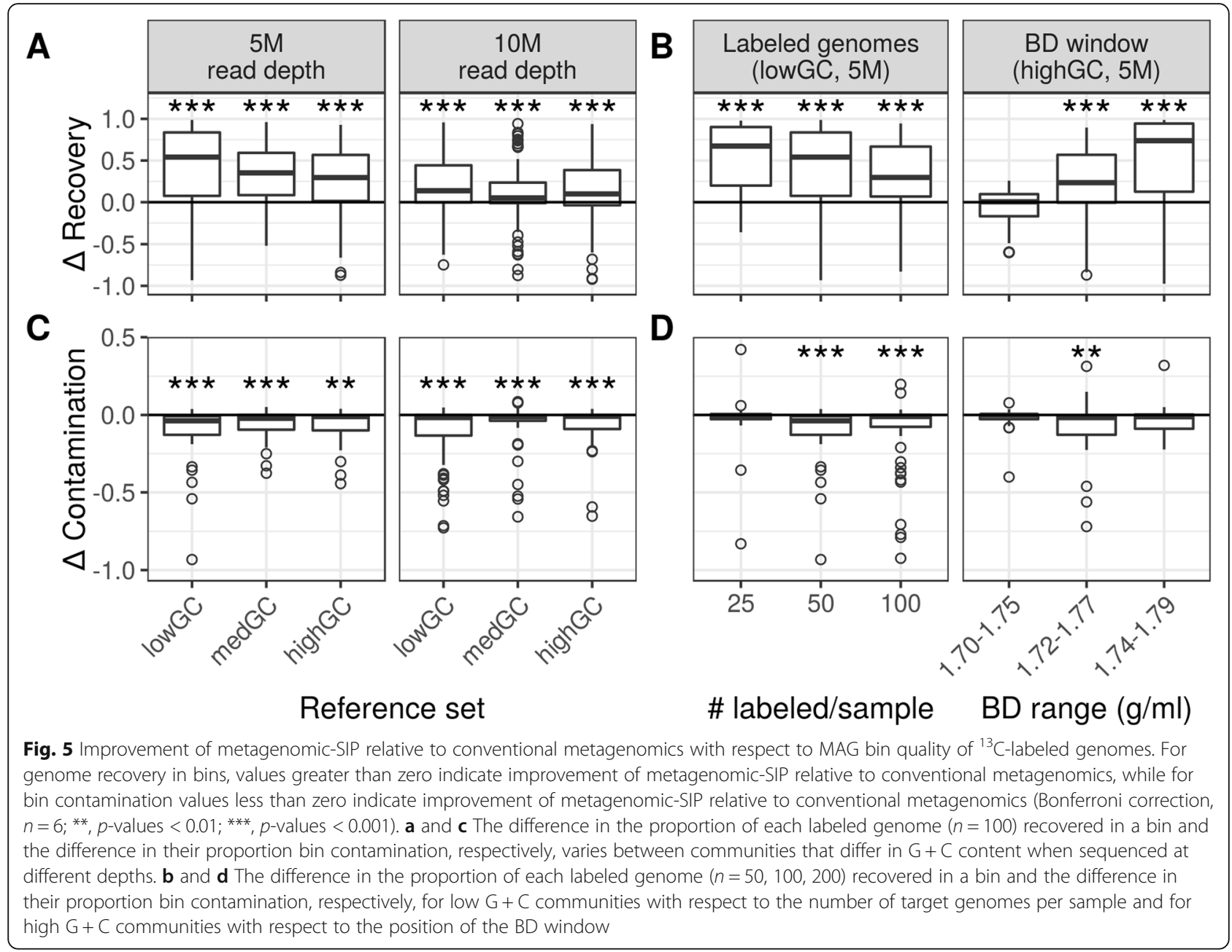

experiments. Further, it may be possible to estimate BD shifts of contigs or MAGs based on theoretical fragment or read distributions generated with MetaSIPSim, thereby enabling quantification of isotopic enrichment.

We used simulated datasets generated with MetaSIPSim to evaluate the efficacy of metagenomic-SIP relative to conventional shotgun metagenomics. In addition to demonstrating the utility of the MetaSIPSim toolkit, this analysis established the power of metagenomic-SIP methodologies. We found that metagenomic-SIP improved the ability to assemble and bin isotopically labeled target genomes with higher quality, greater completeness, and less contamination than could be achieved through the application of conventional shotgun metagenomic sequencing. Examples of these improvements for three individual genomes is shown in Fig. 6. Our analyses confirmed that metagenomic-SIP is an effective method for targeted assembly of genomes from complex metagenomes and helped identify cases where the benefits of this method were marginal. Using MetaSIPSim, researchers can use preliminary knowledge of their system's community composition and the planned experimental parameters to test how well metagenomic-SIP will benefit them and adapt or optimize their sampling, methods, or sequencing regime accordingly.

Our analyses revealed how experimental design influences the power of metagenomic-SIP. We found that the benefits of metagenomic-SIP relative to conventional shotgun metagenomics declined as sequencing depth and genome coverage increased. We believe that this is largely due to the fact that, with deep sequencing, there is enough read coverage to capture all or most of a community and therefore enrichment with stable isotopes is unnecessary. Our simulations were limited to 500 well characterized bacterial genomes, while natural communities, such as soil, contain orders of magnitude more diversity and complexity [45]. Hence, the utility of metagenomic-SIP is likely to be greater with natural communities relative to the simulations we performed. Further, the ability to achieve greater bin quality with less sequencing coverage may make metagenomic-SIP more appealing for studies that require many samples or replicates due to the tradeoff between sequencing depth and sample number [46]. 

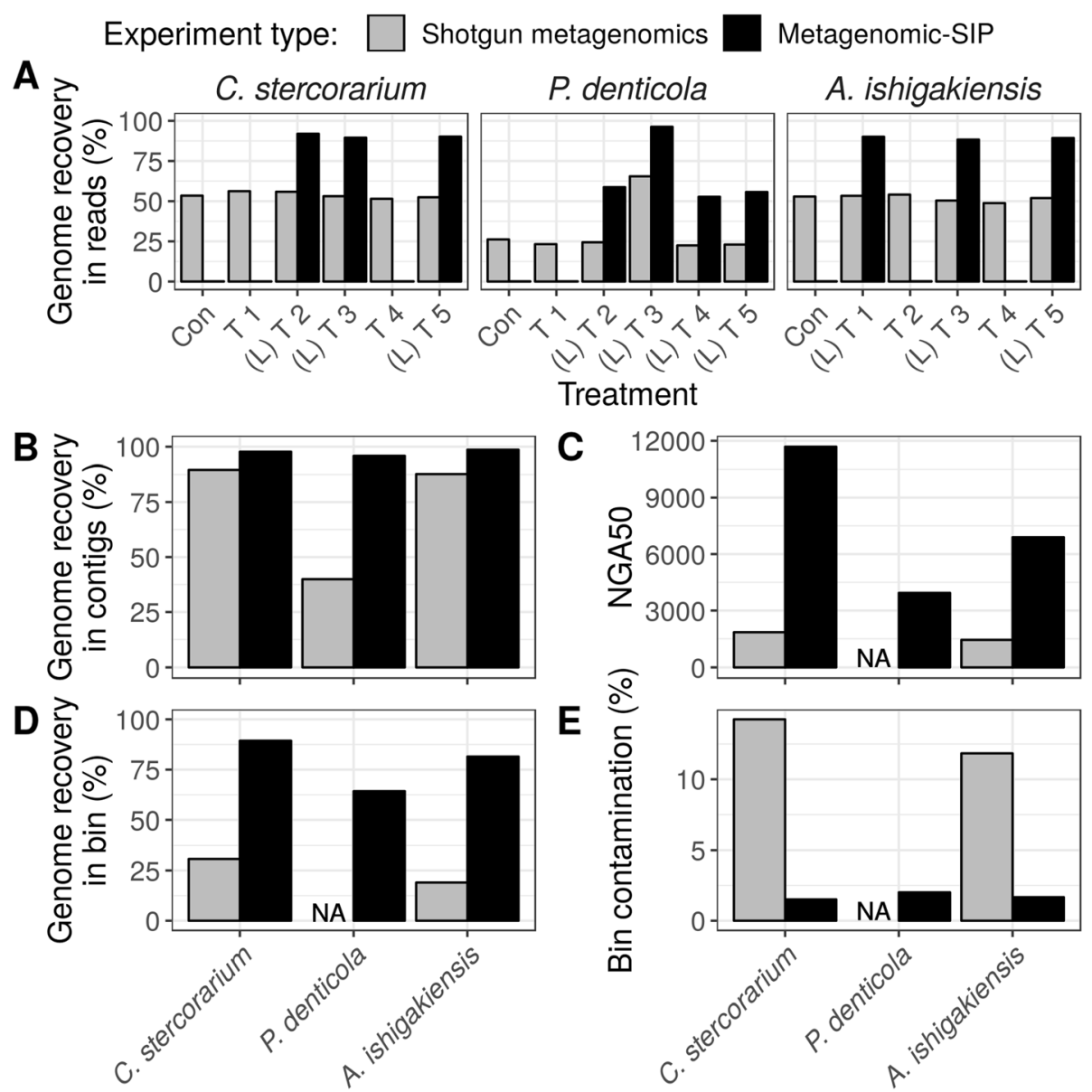

Fig. 6 Examples of MAG quality improvements achieved with metagenomic-SIP relative to conventional shotgun metagenomics for three target genomes of low abundance in the community. These examples are taken from the $50 \% \mathrm{G}+\mathrm{C}$ skewed (medGC) reference set sequenced at 5,000,000 reads. Genomes presented here are Clostridium stercorarium, Prevotella denticola, and Altererythrobacter ishigakiensis. a Percentage of each genome recovered in reads across 6 simulation trials in which community composition and ${ }^{13} \mathrm{C}$-labelling were varied randomly (T1 - T5); 'con' indicates the ${ }^{12} \mathrm{C}$-control, ' $\mathrm{L}$ ' indicates a trail in which the organism was ${ }^{13} \mathrm{C}$-labeled. $\mathbf{b}$ Percentage of each genome recovered in contigs from the co-assembly of all 6 trials. $\mathbf{c}$ The NGA50 of the contigs from the co-assembly mapped to each genome. $\mathbf{d}$ The percentage of each genome recovered in a MAG bin. e The percentage each MAG bin that is contamination from other genomes. Note that we have no NGA50 for the shotgun metagenomics assembly of $P$. denticola as less than $50 \%$ of this genome was recovered from the co-assembly. Similarly, we recovered no bin mapping to $P$. denticola from the shotgun metagenome

The benefits of metagenomic-SIP varied across bacterial communities that differed in $G+C$ skew. Specifically, we saw that metagenomic-SIP improved assembly and binning of incorporators more in the high $\mathrm{G}+\mathrm{C}$ skewed communities than in the lower $\mathrm{G}+\mathrm{C}$ skewed communities. This result is likely due to the incursion of reads from high $\mathrm{G}+\mathrm{C}$ unlabeled genomes, which are naturally recovered in heavy $\mathrm{BD}$ windows. Due to this phenomenon, we recommend a careful selection of the $\mathrm{BD}$ window to be sequenced, based on the overall community $\mathrm{G}+\mathrm{C}$ for metagenomic-SIP studies employing the heavy-SIP method. We confirmed this result by simulating reads from a high $\mathrm{G}+\mathrm{C}$ genome set with both a lighter and heavier $\mathrm{BD}$ window than originally tested. We found that for a community with overall high
$\mathrm{G}+\mathrm{C}$ skew, a heavier BD window resulted in the greatest benefit from metagenomic-SIP.

Regardless of overall community, the benefits of metagenomic-SIP in recovery of an isotopically labeled genome depends on the individual incorporator's $G+C$. Specifically, metagenomic-SIP was less advantageous for recovering with low $\mathrm{G}+\mathrm{C}$ incorporators than for medium to high $\mathrm{G}+\mathrm{C}$ incorporators. Low $\mathrm{G}+\mathrm{C}$ genomes are too light to sufficiently shift into the $\mathrm{BD}$ window that is sequenced, despite isotopic labeling. This loss of low $\mathrm{G}+\mathrm{C}$ genomes may be more extreme if heavier or narrower $\mathrm{BD}$ ranges are chosen. Indeed, we observed this outcome with our follow-up simulation when using a heavier BD window (Additional file 1: Figures $\mathrm{S} 9$ and S15). If a study is designed to specifically 
target low $\mathrm{G}+\mathrm{C}$ genomes, such as some Firmicutes species, a lighter BD window may be optimal for MAG recovery.

Finally, the benefits of metagenomic-SIP were greatest for incorporators present in low abundance in the community. Most highly abundant incorporators had highquality assemblies and bins with both metagenomic methods, yet metagenomic-SIP greatly improved assembly and binning over conventional shotgun metagenomics for lesser abundant incorporators. We conclude that metagenomic-SIP shows great promise for metagenome recovery of very low abundant genomes [21] and have shown examples of this potential with three genomes simulated at low relative abundance (Fig. 6).

For our case study, we simulated a multi-substrate SIP experiment. However, there are a number of other experimental designs popularly used dependent on available resources and study hypotheses. One commonly used simple design is to add a single labeled substrate to single or replicate environmental samples. The goal of this design is to use the isotopic labeling to selectively enrich for labeled genomes and may be useful for identifying slow growing, low abundant organisms that utilize a specific substrate. We predict that this method will perform similarly to the multi-substrate method that we simulated and if sequenced to the same depth, may allow for recovery of even more reads from the target genomes. However, without the variation in coverage due to differential labeling across multiple treatments, assembly and MAG binning may produce poorer results than shown here. Another common metagenomic-SIP experimental design is to use a single isotopically labeled substrate in a time series. This design aims to identify successive changes in active populations utilizing a given substrate and to identify trophic networks. Simulations with this experimental design would be very similar to our analyses. The primary difference being that the user may want to include additional unlabeled control samples, one per timepoint. We have not tested either of these designs here but we encourage using MetaSIPSim to test the benefits of these methods over conventional metagenomics for unique experimental designs and parameters.

\section{Conclusions}

MetaSIPSim is a useful tool for simulation-based testing of metagenomic-SIP methodologies. MetaSIPSim can be used prior to a metagenomic-SIP experiment to optimize experimental parameters, used to develop analytical tools, or used as a component of analysis to identify isotopically labeled genomes. Using MetaSIPSim, we demonstrated that metagenomic-SIP experiments significantly improve assembly and binning of targeted, isotopically labeled genomes. This analysis shows that experimental and community parameters including sequencing depth, BD window selection, community $\mathrm{G}+\mathrm{C}$ content, and number of labeled genomes all significantly influence the benefit of metagenomic-SIP over conventional shotgun metagenomic sequencing.

\section{Availability and requirements \\ Project name: MetaSIPSim \\ Project homepage: https://github.com/seb369/MetaSIPSim Operating system: Linux and Mac OSX \\ Programming language: Python 2.7 \\ Other requirements: See Additional file 1: Table S1 \\ License: MIT license \\ Any restrictions to use by non-academics: None}

\section{Supplementary information}

Supplementary information accompanies this paper at https://doi.org/10. 1186/s12859-020-3372-6.

Additional file 1: Supplementary Materials and Methods. Table S1. MetaSIPSim dependencies. Table S2. Parameters used for the MetaSIPSim validation and case study simulations. * Values may differ between simulations as described in the materials and methods. Table S3. Processing times for all simulations from the cases study. All times are in seconds. Table S4. Summary statistics for recovery of reads, contigs, and MAGs for labeled genomes in initial simulations. Figure S1. Visual description of variables defined in the manuscript and Table 2. Thick blue line indicates the position of a single DNA fragment during and after ultracentrifugation. Centrifugation setup shown here is based on a fixed angled rotor. Figure S2. Diagram of experimental design for case study simulations. Figure S3. RefSeq genomes $\mathrm{G}+\mathrm{C}$ distribution with $\mathrm{G}+\mathrm{C}$ bins rounded to nearest whole number (downloaded January 25, 2019). Figure S4. $G+C$ distributions for the 500 genomes in each reference set. $\mathrm{G}+\mathrm{C}$ bins are rounded to the nearest whole number. Vertical line indicates $\mathrm{G}+\mathrm{C}$ skew of the set. Figure S5. Rank-abundance plots for each simulated sample or replicate community including the ${ }^{12} \mathrm{C}$-control (Con) and all five ${ }^{13} \mathrm{C}$-trials (T1-T5). Random genomes are only labeled in the ${ }^{13} \mathrm{C}$-labeled samples. Vertical grey lines indicate ranks of the 50 labeled genomes per treatment. Labeled genomes were selected such that mean ranks of the labeled genomes averaged across all samples were similar across the reference sets (lowGC, medGC, and highGC). Figure S6. Fold difference in raw read coverage for each labeled genome between the metagenomic-SIP and shotgun metagenomic libraries from the original simulations. Values above one indicate greater coverage in the metagenomic-SIP compared to the shotgun metagenomic libraries. Figure S7. Fold difference in proportion of each labeled genome recovered by reads between the metagenomic-SIP and shotgun metagenomic libraries from the original simulations. Values above one indicate greater recovery in the metagenomic-SIP compared to the shotgun metagenomic libraries. Figure S8. Fold difference in raw read coverage for each labeled genome between the metagenomic-SIP and shotgun metagenomic libraries from the follow-up simulations. Values above one indicate greater coverage in the metagenomic-SIP compared to the shotgun metagenomic libraries. Simulation with the lowGC reference set with varying number of labeled genomes per sample is in the top row while the simulation with the highGC reference set with different sequencing window BD ranges is in the bottom row. Figure S9. Fold difference in proportion of each labeled genome recovered by reads between the metagenomic-SIP and shotgun metagenomic libraries from the follow-up simulations. Values above one indicate greater recovery in the metagenomic-SIP compared to the shotgun metagenomic libraries. Simulation with the lowGC reference set with varying number of labeled genomes per sample is in the top row while the simulation with the highGC reference set with different sequencing window BD ranges is in the bottom row. Figure S10. Difference in proportion of each labeled genome recovered in co-assembled contigs between the metagenomic- 
SIP and shotgun metagenomic libraries from the original simulations. Values above zero indicate greater recovery in the metagenomic-SIP compared to the shotgun metagenomic contigs. Figure S11. Difference in NGA50 of each labeled genome covered by co-assembled contigs between the metagenomic-SIP and shotgun metagenomic libraries from the original simulations. Values above zero indicate greater NGA50 in the metagenomic-SIP compared to the shotgun metagenomic contigs. Only genomes with over 50\% recovery in both SIP and shotgun metagenomes were used in this analysis. Figure S12. Difference in proportion of each labeled genome recovered in co-assembled contigs between the metagenomic-SIP and shotgun metagenomic libraries from the follow-up simulations. Values above zero indicate greater recovery in the metagenomic-SIP compared to the shotgun metagenomic contigs. Simulation with the lowGC reference set with varying number of labeled genomes per sample is in the top row while the simulation with the highGC reference set with different sequencing window $B D$ ranges is in the bottom row. Figure S13. Difference in NGA50 of each labeled genome covered by co-assembled contigs between the metagenomic-SIP and shotgun metagenomic libraries from the original simulations. Values above zero indicate greater NGA50 in the metagenomic-SIP compared to the shotgun metagenomic contigs. Only genomes with over $50 \%$ recovery in both SIP and shotgun metagenomes were used in this analysis. Simulation with the lowGC reference set with varying number of labeled genomes per sample is in the top row while the simulation with the highGC reference set with different sequencing window $B D$ ranges is in the bottom row. Figure S14. Difference in binning quality between the SIP-metagenomes and shotgun metagenomes with the initial simulations using multiple bins per labeled genome. A) Difference in proportion of each labeled genome recovered in bins. B) Difference in the cumulative contamination for each labeled genome bin set. Figure S15. Difference in proportion of each labeled genome recovered in a single most complete bin between the metagenomic-SIP and shotgun metagenomic libraries from the follow-up simulations. Values above zero indicate greater recovery in the metagenomic-SIP compared to the shotgun metagenomic bins. Simulation with the lowGC reference set with varying number of labeled genomes per sample is in the top row while the simulation with the highGC reference set with different sequencing window $\mathrm{BD}$ ranges is in the bottom row.

\section{Abbreviations}

BD: Buoyant density; DBL: Diffusive boundary layer; HR-SIP: High-resolution stable isotope probing; MAG: Metagenome assembled genome; SIP: Stable isotope probing

\section{Acknowledgements}

We thank Nicholas Youngblut for advice and suggestions during development of MetaSIPSim as well as Roland Wilhelm for discussions and review of this manuscript.

\section{Authors' contributions}

DHB conceived of the project, SEB designed and wrote the software and conducted the analysis, SEB wrote the manuscript with input and edits from DHB. All authors have read and approved the final manuscript.

\section{Funding}

This work was supported by the U.S. Department of Energy, Office of Biological \& Environmental Research Genomic Science Program under award numbers DE-SC0016364 and DE-SC0004486. Funding body played no role in study design, collection, analysis, or interpretation of data, or writing the manuscript.

\section{Availability of data and materials}

All reference genomes used in these simulations are available through the NCBI Reference Sequence Database (https://www.ncbi.nlm.nih.gov/refseq/). Code used to generate simulations are available at https://github.com/seb36 9/MetaSIPSim.

Ethics approval and consent to participate Not applicable.

\section{Consent for publication}

Not applicable.

\section{Competing interests}

The authors declare that they have no competing interests.

Received: 13 August 2019 Accepted: 16 January 2020

Published online: 30 January 2020

\section{References}

1. Dumont MG, Murrell JC. Stable isotope probing - linking microbial identity to function. Nat Rev Microbiol. 2005;3(6):499-504. https://doi.org/10.1038/ nrmicro1162.

2. Pepe-Ranney C, Campbell AN, Koechli CN, Berthrong S, Buckley DH. Unearthing the ecology of soil microorganisms using a high resolution DNA-SIP approach to explore cellulose and xylose metabolism in soil. Front Microbiol. 2016;7:703. https://doi.org/10.3389/fmicb.2016.00703.

3. Buckley DH, Huangyutitham V, Hsu S-F, Nelson TA. Stable isotope probing with $15 \mathrm{~N} 2$ reveals novel noncultivated diazotrophs in soil. Appl Environ Microb. 2007;73(10):3196-204. https://doi.org/10.1128/AEM.02610-06.

4. Buckley DH, Huangyutitham V, Hsu S-F, Nelson TA. 15N2-DNA-stable isotope probing of diazotrophic methanotrophs in soil. Soil Biol Biochem. 2008;40(6):1272-83. https://doi.org/10.1016/j.soilbio.2007.05.006.

5. Radajewski S, Ineson P, Parekh NR, Murrell JC. Stable-isotope probing as a tool in Microb Ecol. Nature. 2000;403:646-9. https://doi.org/10.1038/ 35001054.

6. Gupta V, Smemo KA, Yavitt JB, Basiliko N. Active methanotrophs in two contrasting north American peatland ecosystems revealed using DNA-SIP. Microb Ecol. 2012;63(2):438-45. https://doi.org/10.1007/s00248-011-9902-z.

7. Neufeld JD, Schäfer H, Cox MJ, Boden R, McDonald IR, Murrell JC. Stableisotope probing implicates Methylophaga spp and novel Gammaproteobacteria in marine methanol and methylamine metabolism. ISME J. 2007;1(6):480-91. https://doi.org/10.1038/ismej.2007.65.

8. Bryson S, Li Z, Chavez F, Weber PK, Pett-Ridge J, Hettich RL, Pan C, Mayali X, Mueller RS. Phylogenetically conserved resource partitioning in the coastal microbial loop. ISME J. 2017;11(12):2781-92. https://doi.org/10.1038/ismej. 2017.128.

9. Pepe-Ranney C, Koechli C, Potrafka R, Andam C, Eggleston E, Garcia-Pichel F, Buckley DH. Non-cyanobacterial diazotrophs mediate dinitrogen fixation in biological soil crusts during early crust formation. ISME J. 2015;10(2):287-98. https://doi.org/10.1038/ismej.2015.106.

10. DeRito CM, Pumphrey GM, Madsen EL. Use of field-based stable isotope probing to identify adapted populations and track carbon flow through a phenol-degrading soil microbial community. Appl Environ Microb. 2005: 71(12):7858-65. https://doi.org/10.1128/AEM.71.12.7858-7865.2005.

11. Jeon CO, Park W, Padmanabhan P, DeRito C, Snape JR, Madsen EL. Discovery of a bacterium, with distinctive dioxygenase, that is responsible for in situ biodegradation in contaminated sediment. P Natl Acad Sci USA. 2003;100(23):13591-6. https://doi.org/10.1073/pnas.1735529100.

12. Kasai $Y$, Takahata $Y$, Manefield M, Watanabe K. RNA-based stable isotope probing and isolation of anaerobic benzene-degrading bacteria from gasoline-contaminated groundwater. Appl Environ Microb. 2006;72(5):358692. https://doi.org/10.1128/AEM.72.5.3586-3592.2006.

13. Thomas F, Corre E, Cébron A. Stable isotope probing and metagenomics highlight the effect of plants on uncultured phenanthrene-degrading bacterial consortium in polluted soil. ISME J. 2019;13(7):1814-30. https://doi. org/10.1038/s41396-019-0394-z.

14. Uhlik O, Leewis M-C, Strejcek M, Musilova L, Mackova M, Leigh MB, Macek T. Stable isotope probing in the metagenomics era: a bridge towards improved bioremediation. Biotechnol Adv. 2013;31(2):154-65. https://doi. org/10.1016/j.biotechadv.2012.09.003.

15. Shi S, Herman DJ, He Z, Pett-Ridge J, Wu L, Zhou J, Firestone MK. Plant roots alter microbial functional genes supporting root litter decomposition. Soil Biol Biochem. 2018;127:90-9. https://doi.org/10.1016/j.soilbio.2018.09.013.

16. Müller AL, Pelikan C, de Rezende JR, Wasmund K, Putz M, Glombitza C, Kjeldsen KU, Jorgensen BB, Loy A. Bacterial interactions during sequential degradation of cyanobacterial necromass in a sulfidic arctic marine sediment. Environ Microbiol. 2018;20(80):2927-40. https://doi.org/10.1111/ 1462-2920.14297. 
17. Youngblut ND, Barnett SE, Buckley DH. SIPSim: a modeling toolkit to predict accuracy and aid design of DNA-SIP experiments. Front Microbiol. 2018;9: 570. https://doi.org/10.3389/fmicb.2018.00570.

18. Hungate BA, Mau RL, Schwartz E, Caporaso JG, Dijkstra P, van Gestel N, Koch BJ, Liu CM, McHugh TA, Marks JC, Morrissey EM, Price LB. Quantitative microbial ecology through stable isotope probing. Appl Environ Microb. 2015;81(21):7570-81. https://doi.org/10.1128/AEM.02280-15.

19. Grob C, Taubert M, Howat AM, Burns OJ, Dixon JL, Richnow HH, Jehmlich N, von Bergen $\mathrm{M}$, Chen Y, Murrell JC. Combining metagenomics with metaproteomics and stable isotope probing reveals metabolic pathways used by a naturally occurring marine methylotroph. Environ Microbiol. 2015; 17(10):4007-18. https://doi.org/10.1111/1462-2920.12935.

20. Wilhelm RC, Singh R, Eltis LD, Mohn WW. Bacterial contributions to delignification and lignocellulose degradation in forest soils with metagenomic and quantitative stable isotope probing. ISME J. 2019;13(2): 413-29. https://doi.org/10.1038/s41396-018-0279-6

21. Chen Y, Murrell JC. When metagenomics meets stable-isotope probing: progress and perspectives. Trends Microbiol. 2010;18(4):157-63. https://doi. org/10.1016/j.tim.2010.02.002

22. Coyotzi S, Pratscher J, Murrell JC, Neufeld JD. Targeted metagenomics of active microbial populations with stable-isotope probing. Curr Opin Biotech. 2016;41:1-8. https://doi.org/10.1016/j.copbio.2016.02.017.

23. Rolfe $R$, Meselson M. The relative homogeneity of microbial DNA. P Natl Acad Sci USA. 1959;45(7):1039-43. https://doi.org/10.1073/pnas.45.7.1039.

24. Birnie GD, Rickwood D. Centrifugal separations in molecular and cell biology. Boston: Butterworths; 1978.

25. Buckley DH, Huangyutitham V, Hsu S-F, Nelson TA. Stable isotope probing with $15 \mathrm{~N}$ achieved by disentangling the effects of genome $\mathrm{G}+\mathrm{C}$ content and isotope enrichment on DNA density. Appl Environ Microb. 2007;73(10): 3189-95. https://doi.org/10.1128/AEM.02609-06.

26. Schildkraut CL, Marmur J, Doty P. Determination of the base composition of deoxyribonucleic acid from its buoyant density in CsCl. J Mol Biol. 1962;4(6): 430-43. https://doi.org/10.1016/S0022-2836(62)80100-4

27. Schmid CW, Hearst JE. Sedimentation equilibrium of DNA samples heterogeneous in density. Biopolymers. 1972;11(9):1913-8. https://doi.org/ 10.1002/bip.1972.360110911.

28. Ifft JB, Martin WR III, Kinzie K. Density gradient proportionality constants for a number of aqueous binary solutions. Biopolymers. 1970;9(5):597-614. https://doi.org/10.1002/bip.1970.360090505.

29. Clay O, Douady CJ, Carels N, Hughes S, Bucciarelli G, Bernardi G. Using analytical ultracentrifugation to study compositional variation in vertebrate genomes. Eur Biophys J. 2003;32(5):418-26. https://doi.org/10.1007/s00249-003-0294-y.

30. Gourlé H, Karlsson-Lindsjö O, Hayer J, Bongcam-Rudloff E. Simulating Illumina metagenomic data with InSilicoSeq. Bioinformatics. 2019;35(3):5212. https://doi.org/10.1093/bioinformatics/bty630.

31. Frank JA, Pan Y, Tooming-Klunderud A, Eijsink VGH, McHardy AC, Nederbragt AJ, Pope PB. Improved metagenome assemblies and taxonomic binning using long-read circular consensus sequence data. Sci Rep. 2016; 6(1):25373. https://doi.org/10.1038/srep25373.

32. Stewart RD, Auffret MD, Warr A, Walker AW, Roehe R, Watson M. Compendium of 4,941 rumen metagenome-assembled genomes for rumen microbiome biology and enzyme discovery. Nat Biotechnol 2019;37(8):953961. doi:https://doi.org/10.1038/s41587-019-0202-3.

33. Wang B, Qin W, Ren Y, et al. Expansion of Thaumarchaeota habitat range is correlated with horizontal transfer of ATPase operons. ISME J. 2019;13(12): 3067-79. https://doi.org/10.1038/s41396-019-0493-x.

34. Lueders T, Manefield M, Friedrich MW. Enhanced sensitivity of DNA- and rRNA-based stable isotope probing by fractionation and quantitative analysis of isopycnic centrifugation gradients. Environ Microbiol. 2004;6(1): 73-8. https://doi.org/10.1046/j.1462-2920.2003.00536.x.

35. Wawrik B, Callaghan AV, Bronk DA. Use of inorganic and organic nitrogen by Synechococcus spp. and diatoms on the West Florida shelf as measured using stable isotope probing. Appl Environ Microb. 2009;75(21):6662-70. https://doi.org/10.1128/AEM.01002-09.

36. Mitchell M, Muftakhidinov B, Winchen T, Jedrzejewski-Szmek Z, Weingrill J, Langer S, Lane D, Sower K. Engauge Digitizer. 2019. https://markummitchell. github.io/engauge-digitizer

37. O'Leary NA, Wright MW, Brister JR, et al. Reference sequence (RefSeq) database at NCBI: current status, taxonomic expansion, and functional annotation. Nucleic Acids Res. 2016;44(D1):D733-45. https://doi.org/10. 1093/nar/gkv1189.
38. Verastegui Y, Cheng J, Engel K, et al. Multisubstrate Isotope Labeling and Metagenomic Analysis of Active Soil Bacterial Communities. mBio. 2014;5(4): e01157-14. https://doi.org/10.1128/mBio.01157-14

39. Li D, Luo R, Liu C-M, Leung CM, Ting HF, Sadakane K, Yamashita H, Lam TW. MEGAHIT v1.0: A fast and scalable metagenome assembler driven by advanced methodologies and community practices. Methods. 2016;102:311. https://doi.org/10.1016/j.ymeth.2016.02.020.

40. Kang DD, Froula J, Egan R, Wang Z. MetaBAT, an efficient tool for accurately reconstructing single genomes from complex microbial communities. PeerJ. 2015;3:e1165. https://doi.org/10.7717/peerj.1165.

41. Kang D, Li F, Kirton ES, Thomas A, Egan RS, An H, Wang Z. MetaBAT 2: an adaptive binning algorithm for robust and efficient genome reconstruction from metagenome assemblies. PeerJ Preprints. 2019;7:e27522v1. https://doi. org/10.7287/peerj.preprints.27522v1

42. Bushnell B. BBMap short-read aligner, and other bioinformatics tools; 2019.

43. Mikheenko A, Saveliev V, Gurevich A. MetaQUAST: evaluation of metagenome assemblies. Bioinformatics. 2016;32(7):1088-90. https://doi.org/ 10.1093/bioinformatics/btv697.

44. R Core Team. R: A Language and Environment for Statistical Computing. 2018.

45. Roesch LFW, Fulthorpe RR, Riva A, Casella G, Hadwin AK, Kent AD, Daroub SH, Camargo FA, Farmerie WG, Triplett EW. Pyrosequencing enumerates and contrasts soil microbial diversity. ISME J. 2007;1(4):283-90. https://doi.org/10. 1038/ismej.2007.53.

46. Sims D, Sudbery I, llott NE, Heger A, Ponting CP. Sequencing depth and coverage: key considerations in genomic analyses. Nat Rev Genet. 2014; 15(2):121-32. https://doi.org/10.1038/nrg3642.

\section{Publisher's Note}

Springer Nature remains neutral with regard to jurisdictional claims in published maps and institutional affiliations.

Ready to submit your research? Choose BMC and benefit from:

- fast, convenient online submission

- thorough peer review by experienced researchers in your field

- rapid publication on acceptance

- support for research data, including large and complex data types

- gold Open Access which fosters wider collaboration and increased citations

- maximum visibility for your research: over $100 \mathrm{M}$ website views per year

At $\mathrm{BMC}$, research is always in progress.

Learn more biomedcentral.com/submissions 